\title{
Philosophiques
}

\section{Jeux évolutionnaires et paradoxe de l'induction rétrograde (backward induction)}

\section{Pierre Livet}

Volume 25, numéro 2, automne 1998

Les modèles d’évolution en économie et en sciences sociales

URI : https://id.erudit.org/iderudit/027486ar

DOI : https://doi.org/10.7202/027486ar

Aller au sommaire du numéro

Éditeur(s)

Société de philosophie du Québec

ISSN

0316-2923 (imprimé)

1492-1391 (numérique)

Découvrir la revue

Citer cet article

Livet, P. (1998). Jeux évolutionnaires et paradoxe de l'induction rétrograde (backward induction). Philosophiques, 25(2), 181-201.

https://doi.org/10.7202/027486ar
Résumé de l'article

La théorie des jeux évolutionnaires s'oppose à la théorie des jeux classique en ce qu 'elle élimine les raisonnements des joueurs. Peut-elle dépasser les apories (impossibilités de coopérations) de la théorie classique ? Mais en reconsidérant le raisonnement classique d'induction rétrograde, en y introduisant des possibilités de révision, on évite son aspect paradoxal. L'intérêt de la théorie des jeux évolutionnaires est donc surtout de simuler l'évolution d'interactions dans des populations. 


\title{
JEUX ÉVOLUTIONNAIRES et paradoXe de l'induction rít Trograde \\ (backward induction)
}

\author{
PAR \\ Pierre Livet
}

\begin{abstract}
RÉSUMÉ: La théorie des jeux évolutionnaires s'oppose à la théorie des jeux classique en ce qu'elle élimine les raisonnements des joueurs. Peut-elle dépasser les apories (impossibilités de coopérations) de la théorie classique? Mais en reconsidérant le raisonnement classique d'induction rétrograde, en y introduisant des possibilités de révision, on évite son aspect paradoxal. L'intérêt de la théorie des jeux évolutionnaires est donc surtout de simuler l'évolution d'interactions dans des populations.

ABSTRACT: Evolutionary game theory does not take into account reasoning players, in contrast with classical game theory. Can it overcome some drawbacks of classical theory (impossibility of cooperation)? If we reconsider the backward induction reasoning, and if introduce the possibility of revising, paradoxes and drawbacks disappear. So the main interest of evolutionary game theory consists in the simulation of evolution of interactions among populations.
\end{abstract}

Depuis Maynard Smith, la théorie des jeux évolutionnaires s'est bien développée, particulièrement ces dernières années. Dans cette approche, on suppose que des individus d'une population, ou des sous-populations d'une population, sont dotés d'un comportement défini à l'avance et jouent systématiquement une stratégie (une suite d'actions déterminée en réponse à des suites d'actions déterminées des autres membres de la population, quand ils les rencontrent) sans avoir, pour déterminer cette stratégie, à raisonner sur les actions des autres, contrairement à ce qui se passe en théorie des jeux classique. Selon les gains qu'ils retirent de leurs diverses rencontres avec d'autres joueurs jouant soit la même stratégie, soit des stratégies différentes, ils ont ou non la possibilité de se reproduire, dès que ces gains dépassent un certain seuil, si bien que les sous-populations qui correspondent chacune au a gène $»$ d'une stratégie se multiplient, disparaissent ou restent stables. La théorie porte sur ce que peuvent être les régularités de ces évolutions. 
Il s'agit donc d'étudier l'évolution des populations, commandée par leurs interactions. On peut opposer cette approche à la perspective classique de la théorie des jeux, laquelle élabore les principes non pas d'une évolution des populations, mais d'une rationalité interactive. Les justifications des solutions sont alors fondées sur des raisonnements de joueurs, en fonction de ce qu'ils anticipent des actions des autres joueurs, et réciproquement. Alors que les joueurs des jeux évolutionnaires réagissent à chaque rencontre selon la stratégie qui constitue en quelque sorte leur programme, les joueurs classiques raisonnent pour trouver la meilleure stratégie, étant donné les actions des autres joueurs, justifiées elles-mêmes par un raisonnement similaire. Il est clair que si on oppose une approche réactionnelle et une approche raisonneuse, la théorie des jeux évolutionnaires appartient au premier type.

\section{En suivant Binmore}

Ce qui nous intéresse, c'est l'analyse des relations possibles entre les deux approches. On peut suivre Binmore ${ }^{1}$ qui, tout en partant d'une analyse éductive des raisonnements en théorie des jeux (éductive. désigne l'approche qui s'appuie sur les raisonnements des agents asant el pendant la réalisation du jeu), utilise cependant des considérations évolutionnaires. Il note d'abord que selon l'approche: éductive, la complexité se trouve dans les raisonnements des joueurs et la structure de leurs représentations internes, alors que selon l'approche évolutive, les agents sont supposés beaucoup moins complexes, la complexité provenant plutôt de leur environnement (qui est fait d'autres joueurs ou d'autres populations).

Binmore s'est employé à justifier le recours aux jeux évolutionnaires pour mieux comprendre les problèmes de l'approche raisonneuse. Il commence par voir dans l'éduction, censée former le raisonnement, une reproduction et une diffusion de "mêmes ", en empruntant à Dawkins l'analogie entre gènes et représentations'. Il poursuit en imaginant que l'analyse des jeux évolutionnaires porte sur une sorte de compétition entre programmes (chaque programme jouant systématiquement telle stratégie). L'éduction pourrait alors venir d'une semblable évolution, mais à condition qu'elle porte sur des a maîtres programmes " qui ont la capacité de choisir différentes stratégies, parmi une grande variété de jeux différents, et même de choisir des stratégies qui n'ont jamais été jouées auparavant.

$\mathrm{Si}$ on faisait évoluer ces " maîtres programmes" dans une compétition et si on y introduisait deux contraintes, soit une punition pour les décisions prises avec retard et une probabilité plus forte de

1. Ken Binmore, "Modeling Rational Players, part II ", Economics and Philosophy, vol. 4, 1988, p. 9-55.

2. Voir Dan Sperber, La contagion des idées, Odile Jacob, 1996, pour une critique de cette analogie. 
disfonctionnement pour les programmes complexes, alors on pourrait se poser la question de savoir si le concept central de la théorie des jeux, l'équilibre de Nash, reste bien le concept clé.

Ce concept repose sur l'idée suivante : prenons comme donné un coup d'autrui et recherchons quelle est notre meilleure réponse. Réciproquement, prenons cette meilleure réponse comme donnée et cherchons quelle est la meilleure réponse d'autrui. Un équilibre définit (quand il n'y a que deux joueurs et que deux coups possibles pour chacun d'eux) une paire de coups qui sont chacun l'un eu égard à l'autre la meilleure réponse de chacun des deux joueurs. L'équilibre a donc un aspect auto-référent (chaque coup est la meilleure réponse au coup donné par l'équilibre). Par ailleurs, on peut montrer que toute déviation locale hors de l'équilibre mène à un résultat inférieur.

Un des problèmes soulevés par cette idée, c'est qu'il y a souvent plusieurs équilibres de Nash et qu'on ne sait pas comment choisir entre eux. Un autre problème est que certains équilibres de Nash ne sont pas des optimums de Pareto (des états qu'on peut atteindre par un changement qui suscite l'unanimité et tels qu'on ne puisse vouloir en sortir sans qu'au moins un des participants ne s'y oppose). Le troisième problème, qui est lié au second, est que des déviations conjointes peuvent assurer des gains supérieurs aux joueurs (mais ces gains sont toujours sous la menace d'une rupture de la coopération qu'exige la conjonction des déviations). Binmore a renoncé à surmonter les deux derniers problèmes, mais il pense que le premier, celui de la sélection des équilibres, représente un enjeu décisif.

Il ne se satisfait donc pas de l'équilibre de Nash. Il soutient même que ce concept n'est pas si fondamental qu'on pourrait le penser, si on adopte la perspective des jeux évolutionnaires. Plus l'évolution avance, plus souvent les individus appartenant à une sous-population qui dispose d'un maître programme gagnant ont à jouter contre euxmêmes. L'équilibre de Nash repose au contraire sur la confrontation de deux stratégies différentes, il n'est donc pas central.

La notion clé des jeux évolutionnaires se nomme la stratégie évolutionnairement stable (SES). Une stratégie est évolutionnairement stable (1) si, quand elle joue contre elle-même, elle fait au moins aussi bien que toute stratégie concurrente qu'on introduirait contre elle. En effet, si une autre stratégie faisait mieux contre elle et que cette stratégie fût un mutant peu répandu, elle engrangerait surtout le gain majoré qu'elle ferait contre notre stratégie, et donc se répandrait. Mais une fois répandue, elle se rencontrerait alors ellemême fréquemment, et il faudrait alors qu'elle fasse mieux que la stratégie initiale pour l'emporter. (2) Réciproquement, il faut aussi que contre toute stratégie mutante, notre stratégie fasse mieux que cette stratégie mutante contre elle-même. Sinon, quand elle opérerait dans une population mixte des deux stratégies, la stratégie mutante ferait, d'une part, aussi bien que notre stratégie quand elle y serait opposée et, d'autre part, mieux qu'elle quand elle se rencontrerait 
elle-même, si bien que notre stratégie serait envahie. On voit que le résultat du jeu contre soi-même est crucial pour la définition d'une SES.

Dans le scénario imaginé par Binmore, cependant, cette considération n'est pas seule à jouer puisque grâce aux dysfonctions, il y a toujours des programmes qui ne sont pas le programme dominant. Or, leur expansion était peut-être limitée par des programmes que le programme dominant a éliminé et dès lors, ils peuvent devenir performants contre une population débarrassée de leur's adversaires et composée très majoritairement du programme qui a éliminé ces adversaires. Quoi qu'il en soit, Binmore soutient que nous devrions interpréter la notion d'équilibre en terme d'équilibre évolutionnairement stable plutôt que comme équilibre de Nash.

En 1993, Binmore revient à la charge en rappelant que les jeux évolutionnaires pouvaient apporter une solution au problème de sélection des équilibres ${ }^{3}$. Puis, en 1994, dans son ouvrage Playing Fair ${ }^{4}$, il suggère que cette sélection pourrait nous faire passer du c jeu de la vie ", qui produit une société dont la structure tient à l'histoire du jeu et est donc largement contingente, au « jeu de la morale », qui consiste justement à opérer des sélections entre les sociétés produites par les différents jeux de la vie, donc entre les différents équilibres. Enfin, dans un autre texte de $1993^{5}$, il soutient que réduire les procédures de révision des probabilités à la procédure bayésienne (classique en probabilités) ne marche que dans un univers fermé, alors que toutes nos procédures effectives de décision se déroulent dans un univers ouvert, sans que nous puissions prétendre prévoir tous les possibles. Évidemment, l'univers des jeux évolutionnaires est aussi un univers fermé (puisque toutes les stratégies sont définies d'avance). Mais comme imaginer tous les possibles à l'avance pour définir un équilibre de Nash dans un univers d'une telle complexité ne nous sert de rien, puisque nous utiliserons forcément des approches incomplètes et moins sophistiquées, les jeux évolutionnaires dont les joueurs ne raisonnent même pas et se bornent à réagir peuvent être plus instructifs quion ne le croyait. Leur fermeture n'a pas d'importance si leurs joueurs n'ont rien à anticiper et la richesse des possibilités évolutionnistes donne par ailleurs une idée plus repérable de la richesse encore supérieure que donneraient des jeux dont les agents seraient sophistiqués. Davantage, au lieu de rechercher la meilleure règle de révision possible (ce qui est impossible dans un environnement ouvert, puisqu'on est alors dans une situation où le problème de l'arrêt d'une

3. Ken Binmore, " Rationalizing Backward Induction ", Conference on Rationality in Economics, Turin, octobre 1993.

4. Ken Binmore, Playing Fair, I, Cambridge, MIT Press, 1994.

5. Ken Binmore, "De-Bayesing Game Theory ", dans K. Binmore, Alan Kirman, Piero Tani, Frontiers of Game Theory, Cambridge, MIT Press, 1993, p. 321-339. 
Machine de Turing sur une meilleure solution est impossible à résoudre), il vaut mieux étudier l'émergence de telle ou telle règle d'apprentissage selon les différentes pressions évolutives. Ainsi, alors que les jeux évolutionnaires semblaient nous donner au mieux des simulations d'automates bornés, bien dépassés par les raisonnements sophistiqués de joueurs capables de révisions bayésiennes (dans des jeux à information imparfaite), ils nous permettraient en fait de mieux comprendre l'émergence de règles d'apprentissage autres que les règles bayésiennes et mieux adaptées à des univers ouverts.

Binmore fait donc grand cas d'un résultat de L. Samuelson ${ }^{6}$, qui a calculé quelles stratégies étaient éliminées dans les jeux évolutionnaires. On a en effet proposé de fonder le raisonnement qui, en théorie des jeux, justifie un équilibre sur une procédure d'élimination des stratégies dominées', et même des faiblement dominées, procédure menée de manière récurrente (on reprend le sous-jeu qui reste une fois éliminées ces stratégies et on recommence l'élimination, si elle est possible). Or, Samuelson montre qu'en général, la dynamique des jeux évolutionnaires ne conduit pas à cette élimination. Il faut, pour y arriver, introduire un processus de main tremblante qui introduit un peu d'aléatoire entre les stratégies choisies et les stratégies effectivement jouées. Cette introduction d'aléas doit, pour être efficace, donner des tremblements arbitrairement plus amples que ne le sont déjà les mutations qui font partie du jeu évolutionnaire. Dès lors, ce processus apparaît ad hoc, dédié à l'élimination des stratégies faiblement dominées et on ne peut plus utiliser la main tremblante comme une justification de la procédure d'élimination. Or, l'idée de main tremblante était un prolongement d'une idée de Nash : regardons ce qui se passe si on s'autorise de légères déviations (celles que pourrait introduire un léger aléa) et nolons vers quelle solution elles nous font revenir. Mais ici les déviations devraient être calculées tout exprès.

Selon Binmore, Kirman et Tani, on ne peut pas s'attendre à ce que l'évolution mime les démarches et les résultats de l'introspection raisonneuse. On pourrait en conclure que jeux évolutionnaires et approche raisonneuse peuvent se développer chacun de leur côté. Mais Binmore (1993) va plus loin. Pour lui, l'approche raisonneuse conduit à des paradoxes que l'approche évolutionnaire évite (puisqu'elle est seulement réactive). Le paradoxe en question est celui que semble présenter le raisonnement de backward induction ou induction rétrograde. Ce raisonnement consiste à partir du dernier noeud du jeu (ou des derniers nouds), c'est-à-dire du dernier choix de coups offert au dernier joueur. Le joueur y choisit le coup qui

6. Larry Samuelson, "Does Evolution Eliminate Dominated Strategies? ", dans K. Binmore, A. Kirman, P. Tani, Frontiers of Game Theory.

7. Une stratégie est dominée s'il existe une autre stratégie qui fait mieux qu'elle contre toutes les stratégies adverses; elle est faiblement dominée si cette autre stratégie fait soit mieux soit aussi bien qu'elle. 
maximise son gain. On rétrograde alors au nœud précédent, où le joueur dont c'est le tour de choisir (un autre joueur, généralement) sait maintenant que choisir le coup qui conduit à tel nœud, c'est obtenir le gain que lui fixe le choix du dernier joueur. Il choisit en fonction de cette anticipation, toujours en maximisant. On réitère le processus (d'où le nom d'induction, en référence à l'induction par récurrence sur les nombres et la fonction successeur) jusqu'à parvenir au premier nœud, où l'on découvre alors quelle suite de coups a été sélectionnée.

D'où vient le problème? Les anticipations des joueurs sont fondées seulement si le coup anticipé est celui d'un joutur rationnel (qui maximise). Mais supposons qu'un joneur puisse jouer deux fois, une au premier noud, une au troisième, encadrant ainsi l'unique noud de choix du deuxième joueur. Supposons que le raisonnement rétrograde lui enjoigne de quitter le jeu au premier coup. Comment le second joueur peut-il alors considérer comme rationnel le joueur en question, puisqu'il lui faut, pour raisonner, supposer que ce joueur étrange joue au troisième nœud, ce qui implique qu'il n'a pas quitté le jeu au premier nœud?

Binmore pense que ce paradoxe ne peut être évité. Or, il tient évidemment à ce qu'on dote les joueurs de capacités de raisonner en fonction de ce qu'ils anticipent des raisonnements des autres. Le paradoxe de l'induction rétrograde semble donc constitutif de l'approche raisonneuse. Par ailleurs, ce paradoxe est aussi lié au découpage du raisonnement en plusieurs étapes qui se révèlent emboîtées les unes dans les autres. Cela jette donc un doute sur la procédure d'élimination itérative des stratégies dominées, qui procède à de semblables découpages des emboîtements. Binmore justifiait sa méfiance sur ce dernier point en notant que si on introduit du bruit dans une population donnée (mais pas dans une autre) dans un jeu évolutionnaire, alors il se peut que cela crée une pression non pas en faveur, mais contre l'équilibre de sous-jeu parfait (défini comme celui dont tous les coups sont des solutions en tout sous-jeu, c'est-à-dire dans les jeux qu'on obtient en ne retenant d'abord que les derniers rameaux de l'arbre du jeu, puis les sousbranches et les branches). Dans ce cas, des stratégies faiblement dominées ne seront pas éliminées. Le résultat de Samuelson lui a donné raison.

\section{Analyse des jeux évolutionnaires}

Notons cependant que ce plaidoyer de Binmore en faveur des jeux évolutionnaires est quelque peu indirect. Il y trouve surtout des possibilités d'éviter certaines difficultés de la théorie des jeux classique. Il nous faut donc examiner de manière plus directe les intérêts de l'approche évolutionnaire.

Les partisans des jeux évolutionnaires, cependant, sur les trois raisons qu'ils nous proposent de nous passionner pour les jeux 
évolutionnaires, n'en donnent qu'une qui soit directe, et deux indirectes.

Commençons par les raisons indirectes, autour desquelles ont déjà tourné les réflexions de Binmore. La première, c'est que la notion d'équilibre de Nash n'est pas liée à une quelconque procédure qui parviendrait à cet équilibre. Un équilibre de Nash, on l'a vu, est simplement la meilleure réponse que chacun puisse faire à un coup de l'autre supposé donné, en supposant qu'on doive ensuite échanger les perspectives, si bien que le coup de l'autre sera aussi choisi comme meilleure réponse au précédent supposé donné. Une fois un coup supposé donné, l'équilibre de Nash s'obtient quand on nous redonne ce coup - une fois permutés les points de vue - comme meilleure réponse. Il constitue donc un point fixe (qu'on définit comme la valeur pour laquelle la fonction qui opère sur cette valeur redonne la même valeur). Mais la plupart du temps, on ne sait pas comment parvenir à un point fixe. Il est donc intéressant de calculer des cheminements dans un jeu, en se permettant des déviations et en regardant si ces déviations se corrigent d'elles-mêmes, et comment on parvient à une solution. On se demande donc si la dynamique que l'on peut calculer donnera ou non tel type d'équilibre. On souhaite en principe que l'évolution révèle l'équilibre de Nash comme stratégie évolutionnairement stable. On souhaite aussi que, lorsqu'il existe plusieurs équilibres de Nash, l'évolution fasse son travail de sélection et nous en recommande un.

La seconde raison, c'est que les raisonnements qu'on doit supposer aux agents de la théorie des jeux deviennent, si on introduit des considérations de logique épistémiques, extrêmement sophistiqués (ils exigent le savoir commun de la rationalité, donc de savoir que je sais que vous savez que je sais, etc. à l'infini). Ceci permet aux agents de raisonner à des niveaux de raisonnement emboîtés infinis, ce qui semble très loin des pratiques humaines. On a donc peu d'espoir qu'une approche en théorie des jeux puisse prédire quelque comportement que ce soit. De fait, les expérimentations psychologiques montrent que nous ne nous comportons pas comme la théorie des jeux souhaiterait que nous le fassions. Or, les agents des jeux évolutionnaires sont beaucoup moins sophistiqués, puisqu'ils ne font aucun raisonnement. On peut donc espérer que les stratégies qui y émergent seront plus proches des stratégies pratiquées. Notons que cette justification de praticabilité peut avoir des buts opposés à la première. En effet, on souhaiterait ici obtenir non pas uniquement des équilibres de Nash, mais aussi des situations paretiennes (par exemple, la coopération dans le dilemme du prisonnier, qui n'est pas un équilibre de Nash).

On peut rattacher à cette seconde justification un argument moins fondamental, mais qui joue un rôle dans l'attraction qu'exercent les jeux évolutionnaires : l'analogie avec le modèle évolutionnaire donne une allure de plausibilité biologique à une 
théorie à laquelle on reprochait d'être très loin d'une bonne modélisation des interactions humaines.

La troisième raison est la seule directe. C'est que l'étude des dynamiques des stratégies et de l'évolution de leur répartition au sein d'une population est en elle-même intéressante. Elle permet de tenir compte à la fois de la diversité populationnelle environnementale et le la diversité historique des situations populationnelles. Pluralité environnementale : une stratégie peut se maintenir dans l'environnement d'autres stratégies favorables (elle-même, la plupart du temps) ; inversement, telle stratégie agressive s'élimine quand elle est opposée à des stratégies tout aussi agressives qu'elle. Diversité historique : le résultat final, dans les jeux évolutionnaires, dépend souvent de la distribution initiale des stratégies dans la population grolale; il est possible, par exemple, qu'une stratégie 2 soit gagnante danis un premier temps étant donné la population 1 de départ, puis que, sa sous-population grandissant, elle ouvre la voie à une stratégie mutante 3 qui exploite la stratégie 2 et qu'ensuite le même phénomène se reproduise, la croissance de la population 3 se trouvant envahissable par une dernière stratégie qui se trouve être la stratégie initiale $^{8}$. Les jeux évolutionnaires nous offrent donc un riche éventail de dynamiques possibles, ils font entrer la théorie des jeux dans l'ère de la simulation de la complexité.

Les résultats de la théorie des jeux évolutionnaires justifient-ils ces espoirs?

Considérons la première justification, soit lintroduction d'un cheminement vers l'équilibre et la sélection des équilibres. Les raisonnements vont s'appuyer ici sur des systèmes d'équations différentielles chargés de modéliser diverses dynamiques. La troisième raison se noue donc avec la première. Par conséquent, on ne s'en tient pas à la notion de point fixe qui semble être le fondement de l'équilibre de Nash (si on prend un profil de stratégies, c'est-à-dire l'ensemble constitué d'une stratégie par' agent, l'équilibre de Nash est la meilleure réplique à lui-même, c'est donc un point fixe de la correspondance qui définit la meilleure réplique en stratégies mixtes ${ }^{9}$. On envisage bien des déviations hors de cet équilibre, ainsi qu'une dynamique qui ramène à l'équilibre.

À cet égard, les deux principales notions utilisées sont aisément différenciables. La stabilité de Lyapounov garantit seulement que si une petite déviation se produit. elle ne va pas nous entraîner loin de l'équilibre. Si bien qüil est possible que plusieurs petites déviations cumulées nous entraînent loin de l'équilibre, car il nyy a pas de force de rappel pour nous y ramener ${ }^{10}$. La stabilité asymptotique, en

8. Jörgen W. Weibull, Evolutionary Game Theory; Cambridge, MIT Press, 1995, p. 90.

9. Une stratégie mixte est une stratégie que l'on joue avec une certaine probabilité, décidée à l'avance en fonction du jeu.

10. Ibid., p. 93. 
revanche, fournit une force de rappel qui ramène à l'équilibre si une déviation se produit ${ }^{11}$. La théorie des stratégies évolutionnairement stables, qui est liée à la stabilité de Lyapounov, n'explique donc pas comment une population finale peut arriver à manifester telle ou telle stratégie. Elle explique seulement comment une fois atteinte, cette stratégie est robuste (dans une mesure limitée, cependant). André Orléan note même que les critères de stabilité (y compris asymptotique) a n'exploitent que très peu le cadre dynamique que proposent les jeux évolutionnistes; seul ce qui se passe au voisinage: des équilibres est pris en compte ${ }^{12}$ ». Il préfère le concept de stabilité stochastique, introduit par Foster et Young, qui, au lieu de considérer comme dans les SES des perturbations mutationnelles ponctuelles et isolées, imaginent des chocs continuels, qui peuvent opérer en série. Ce critère semble sélectionner les mêmes équilibres que celui de la stabilité asymptotique.

Les dynamiques des jeux évolutionnaires peuvent-elles opérer une sélection parmi les équilibres de Nash ? On a montré que dans une dynamique dite de réplicateurs (c'est-à-dire où la reproduction a lieu dans un temps continu, et non pas à des périodes données), les stratégies strictement dominées et aussi les stratégies strictement dominées itérativement sont éliminées. Mais ce n'est pas le cas si la dynamique est discrète ${ }^{13}$. Et cela n'est pas vrai pour toutes les stratégies faiblement dominées ${ }^{14}$. Les états stationnaires non instables dans cette dynamique continue sont ceux qui correspondent au comportement d'équilibre de Nash agrégét ${ }^{15}$. De plus, certains des équilibres de Nash sont instables et ne survivent, sous le critère de stabilité asymptotique, que les équilibres de Nash parfaits (i.e. ceux qui résistent à une modification de main tremblante, proposée par Selten en 1975, et qui consiste, comme on l'a vu, à supposer que les joueurs se trompent parfois, aléatoirement, dans les coups qu'ils jouent), ce qui semble une conséquence logique du critère de stabilité asymptotique.

La sélection la plus drastique est obtenue par les dynamiques à multipopulations. On souhaite introduire des interactions non symétriques entre les joueurs et, pour ce faire, on tire au hasard les individus des sous-populations, au lieu de faire se rencontrer systématiquement les individus de chaque sous-population deux à deux. Du coup, un mutant ne se rencontre jamais lui-même, car dès le moment où il est tiré, il rencontre tous les représentants des autres sous-populations ${ }^{16}$. Dans cette dynamique, ce sont seulement les

11. Ibid., p. 243.

12. André Orléan, "De la stabilité évolutionniste à la stabilité stochastique, réflexions sur les jeux évolutionnistes stochastiques", dans Revue Économique, numéro spécial AFSE, 1996.

13. Ibid., p. 121.

14. Ibid, p. 79 et L. Samuelson, "Does Evolution Eliminate Dominated Strategies? ".

15. J. W. Weibull, Evolutionary Game Theor;.p. 70.

16. Ibid., p. 163. 
équilibres de Nash stricts (pas ceux entre lesquels les joueurs pourraient être indifférents) qui sont asymptotiquement stables. Cela dépend cependant de la dynamique des gains et l'on peut trouver des évolutions de gains en fonction de la diffusion de la population (évolutions cependant croissantes et monotones, mais pas linéaires) qui permettent à des stratégies strictement dominées de survivre ${ }^{17}$ ! Mais le problème est que les dynamiques qui opèrent ces restrictions en opèrent trop : elles éliminent les profils de stratégie mixtes qui tirent au sort les coups à jouer en respectant une certaine probabilité. Il suffit donc qu'un seul agent utilise une stratégie mixte pour que le $n$-uplet de stratégie soit éliminé. Or, il est des jeux qui n'ont pas de solution en stratégies pures (sans tirage probabiliste), mais seulement en stratégies mixtes.

De plus, il n'y a pas cohérence entre les SES et les stratégies atteintes par la dynamique dite du réplicateur: une stratégie $\mathrm{x}$ peut ne pas être une SES et cependant être asymptotiquement stable parce qu'elle bénéficie de la concurrence entre deux autres stratégies $y$ et $z ; x$ est vulnérable à $y$, mais $y$ est vulnérable à $z$, si bien qu’on observe une évolution en spirale qui s'éloigne souvent de $\mathrm{x}$, mais finit par y aboutir ${ }^{18}$.

Les dynamiques des jeux évolutionnaires peuvent-elles donc justifier la déclaration satisfaite de Weibull : « la stabilité dynamique dans la dynamique des réplicateurs, alors même qu'elle est obtenue sans la moindre hypothèse de rationalité, implique un comportement agrégé qui est “ rationnel " et "coordonné" dans le sens de l'équilibre de $\mathrm{Nash}^{19}$ "? On aurait ainsi fait l'économie des hypothèses sur la rationalité des joueurs et sur leur connaissance de cette rationalité, dont le statut logique est soit incertain, soit peu plausible (ainsi l'introspection négative: si je ne sais pas telle proposition, je sais que je ne la sais pas), et il aurait suffit pour cela de satisfaire le critère de stabilité asymptotique. On a vu qu'en fait la sélection des équilibres de Nash ne suivait pas tout à fait une progression conforme à la normativité "rationnelle ". Elle ne permettait pas d'éliminer les uns après les autres les non-solutions, puis les équilibres, selon les catégories emboîtées proposées par l'analyse "raisonneuse ». En effet, les équilibres éliminés le sont en fonction des spécificités des stipulations de la dynamique choisie et il n'y a pas de raison a priori, ni a posteriori, pour que l'on puisse définir une classe de modifications de ces stipulations qui soit strictement parallèle aux exigences de plus en plus serrées de l'analyse "raisonneuse ". Ce qu'on observe plutôt, c'est que tantôt ces stipulations vont effectivement converger vers des réquisits "rationnels» plus exigeants, tantôt elles vont avoir un effet orthogonal, sans rapport avec ces exigences, et vont donc bien opérer

17. Ibid., p. 202 ; exemple dû à Samuelson et Zhang, et utilisé par Binmore.

18. Ibid., p. 102.

19. Ibid., p. 70. 
des sélections, mais qui éliminent des équilibres souhaités par l'analyse a raisonneuse „ et qui en conservent d'autres que celle-ci n'avait pas retenus comme solutions.

Considérons maintenant la deuxième justification, et d'abord l'analogie avec l'évolution. Ici, plusieurs problèmes se posent. Si on admet le théorème de Fisher (1930), dans certains contextes l'évolution induit une croissance monotone avec le temps de la fitness de la moyenne de la population. L'équivalent de la fitness est ici le gain cumulé qu'on peut attribuer à la moyenne de la population. Or, il se révèle que seuls les équilibres de Nash stricts sont évolutionnairement stables quand on utilise le meilleur critère (le plus robuste), celui de la stabilité asymptotique. Des situations paretiennes sont donc éliminées dans certains cas, si bien que le gain de la population survivante est inférieur à celui qu'obtenaient certaines populations éliminées (celles qui pratiquaient plus de coopération) quand elles étaient suffisamment nombreuses. Les modèles des jeux évolutionnaires sont par ailleurs plus ou moins fidèles à l'analogie darwinienne. Par exemple, la dynamique du réplicateur n’implique aucune mutation, mais simplement la présence de populations différentes, alors que la notion de stratégie évolutionnairement stable (SES) est définie comme la résistance à l'invasion par une petite population de mutants (au-dessous d'un certain seuil). Le problème, on l'a vu, c'est que la notion de SES, qui est plus fidèle à l'analogie néodarwinienne que la dynamique du réplicateur, sur ce point, ne satisfait pas à la première justification : donner l'histoire d'une dynamique comme explication de l'émergence d'un équilibre de Nash. De plus, la dynamique qui mène aux SES implique des rencontres qui sont des interactions symétriques deux par deux, ce qui n'est guère plausible dans une véritable évolution. On pourrait dire aussi que cette dynamique exige que les mutants soient rares (audessous de la barrière de résistance, i.e. d'une certaine proportion de mutants) et laissent le temps à la population de s'ajuster en retour avant que n'intervienne la mutation suivante ${ }^{20}$. La dynamique multipopulationnelle évite certains de ces biais en faisant jouer un mutant contre tous les représentants des autres sous-populations, mais elle suppose inversement qu'un mutant ne se rencontre pas luimême, ce qui n'est plausible qu'à l'origine de la mutation.

Envisageons maintenant, dans cette seconde justification, le souhait d'être plus proche des pratiques sociales que les " raisonneurs ». Weibull note une analogie entre une SES et une convention à la Lewis. Ce logicien imagine qu'entre plusieurs règles de conduites possibles, on va choisir celle dont on pense que si tous les autres la choisissent, on a intérêt à la choisir, que les autres pensent de même et que tout cela, chacun le sait, chacun sait que les autres le savent, etc. C'est alors une a convention ». Or, les individus 
qui utilisent la stratégie dominante d'un jeu évolutionnaire n'ont aucune incitation à en changer et les autres, après leur expérience malheureuse, ont intérêt à revenir à cette pratique à laquelle tous se conforment. On pourrait donc donner sens à la notion de convention sans avoir recours à l'appareillage lourd du savoir commun et de ses raisonnements sur les raisonnements d'autrui, tel que proposé par Lewis. Le problème ici, c'est que les conventions sont souvent, sinon toujours, liées à des représentations. Or, il est possible de développer des représentations de manière virtuelle, sans encore leur donner de suite active, et cependant de diffuser ces représentations en les exprimant, avant même qu'elles aient des effets sur les comportements. Une mutation qui est dictée par des représentations peut donc se déclarer brusquement et se diffuser sans incidences pratiques, si bien qu'elle n'a pas à combattre les pratiques auxquelles elle s'oppose. Il se peut même qu'une représentation soit obtenue par des inférences que chacun peut faire en son for intérieur, sans avoir eu besoin de l'exprimer explicitement., et qu'une fois ces inférences parvenues à leur conclusion, la représentation se mette à dominer les pratiques. Mais alors, on n'a pas affaire à une invasion de quelques mutants isolés, mais à un soudain basculement d'une fraction considérable de la population. Ni la notion de SES, ni les dynamiques utilisées habituellement ne permettent de traiter ce problème. Les dynamiques d'imitation analysées par André Orléan vont dans cette voie et montrent la possibilité d'une multiplicité d'états stationnaires, mais elles supposent toujours des transitions continues, même si elles peuvent être brutales.

Les théoriciens des jeux évolutionnaires ont tenté d'introduire un germe d'activité cognitive dans leurs schémas. Ainsi, ils ont envisagé la possibilité de jeux de cheap talk, de communication sans coû́t, chaque individu pouvant passer un message aux autres et utiliser les messages reçus pour déclencher une règle de décision qui prescrit l'action à accomplir. Ou encore, ils introduisent un individu qui est un homo oconomicus extralucide, qui reconnaît les types des agents en face de lui et joue en fonction de leur stratégie (face à un autre homo oconomicus, il joue la stratégie pure non strictement dominée itérativement). Dans ce dernier cas, on peut observer des évolutions qui dépendent de la population initiale et où les trois stratégies pures du jeu pris pour exemple sont toutes utilisées dans diverses proportions ${ }^{21}$. Mais cet agent extralucide est de nouveau peu plausible cognitivement. Nous n'avons pas la capacité de deviner le type de nos adversaires et nous ne pouvons pas l'avoir. En effet, la dissimulation est une conduite cognitive qui est efficace au moins à la première rencontre et qui n'est percée à jour qu'à long terme. On peut donc conclure que si les jeux "raisonneurs " le sont peut-être trop par rapport à la pratique sociale, les jeux évolutionnaires ne

21. Ibid., p. 131. 
tiennent pas compte de toutes les possibilités cognitives des agents. La capacité de cognition va de pair avec la capacité de réviser ses représentations, et ce n'est pas le cas des «animats " des jeux évolutionnaires.

\section{L'« induction rétrograde » est-elle paradoxale ?}

Revenons à présent aux relations entre le souhait d'échapper au paradoxe de la backward induction et le recours à l'approche évolutionnaire. Il faut d'abord noter que l'approche évolutionnaire se développe à partir des jeux sous forme normale (on représente le jeu par une matrice, indiquant pour chaque case qui croise un coup d'un joueur et la réponse des autres le gain de chaque joueur), alors que le paradoxe en question naît d'une analyse des jeux sous forme extensive (on représente le jeu par l'arbre des coups qui se succèdent). Comment fonder ma décision sur l'anticipation d'un coup supposé rationnel auquel autrui ne parviendra jamais s'il est rationnel au premier coup? Aumann a contourné le problème en séparant chaque joueur en ses "avatars " en chaque nœud, ce qui lui permet de faire le raisonnement sur chaque sous-jeu, en admettant la rationalité de chaque sous-agent. Mais cela exige de ne jamais tenir compte du passé (c'est au sous-agent qui intervient une étape plus tôt dans le raisonnement rétrograde de prendre en comple ce passé que son coup constitue, mais il le prend évidemment en compte au présent). Binmore veut utiliser ce " paradoxe " pour récuser la notion d'équilibre de Nash et propose de remplacer l'approche raisonneuse par l'introduction d'un point de vue évolutionnaire dans l'éduction qui nous permettrait de sélectionner nos raisonnements mêmes.

Je ne ferai qu'esquisser une position assez différente. Tout d'abord, il n'y a pas de réel paradoxe d'induction rétrograde, ou plutôt il n'existe que si on découpe le jeux en tranches pour faire le raisonnement. Mais cela n'est pas nécessaire. Il est évidemment nécessaire de partir des derniers nœuds du jeu pour raisonner, car si l'on partait du début, on devrait attendre d'avoir justifié les suites de coups choisies jusqu'aux derniers nœuds pour pouvoir raisonner. Mais partir des derniers nœuds n'implique pas d'être aveugle aux coups précédents. Le raisonnement à faire est un raisonnement par l'absurde.

Prenons, pour le montrer, le jeu de Reny. C'est un jeu séquentiel. Les joueurs jouent l'un après l'autre. Mais ils doivent décider leur stratégie par avance (mettons qu'ils l'écrivent et la confient à un arbitre) et l'exécuter ensuite sans pouvoir la changer. Pierre peut quitter au noud 1 -. il obtient alors 1 et Marie 0 - ou continuer. Marie au nœud 2 peut alors quitter - et obtenir 3 et Pierre 0 - ou continuer. Enfin au noud 3, Pierre peut coopérer - Marie gagne alors 5 et Pierre 2 - ou ne pas coopérer - et il gagne alors 3 et Marie 1 (les gains sont là pour fixer les idées, seul compte leur ordre respectif). 


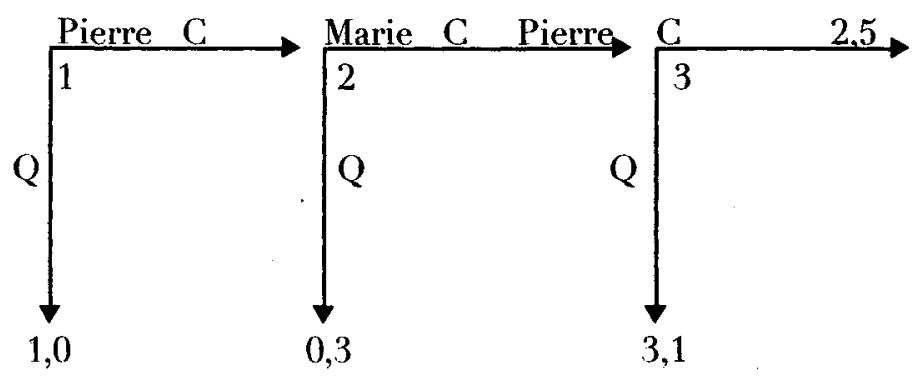

Supposons par hypothèse que nous soyons au nœud 3. Notre raisonnement conditionnel a d'abord pour antécédents les coups hypothétiques passés (Pierre a continué en 1, Marie a continué en 2). Il a ainsi des antécédents au passé et une conclusion au présent. La décision maximisatrice de Pierre en 3 est de ne pas coopérer. II nous faut prendre maintenant ce coup hypothétique pour un antécédent d'un nouveau conditionnel, qui est un conditionnel anticipant. Son antécédent est donc au futur et sa conclusion, au présent. En supposant que Pierre jouera " ne pas coopérer en 3 n, est-ce que Marie joue bien encore " continuer en 2 »? Non, elle joue \& quitter en 2 ». Dès lors, notre précédent conditionnel (passé-présent) est en contradiction avec notre conditionnel en cours (futur-présent). Cependant, la contradiction pourrait simplement venir du coup de Marie, mais pas de celui de Pierre en 1. Il faut alors évaluer un nouveau conditionnel futur-présent. À supposer que Marie quitte en 2, est-ce que Pierre continue en 1 ? Non, il quitte et on a, là encore, une contradiction. Notre hypothèse initiale était donc contradictoire et la seule qui ne le sera pas, c'est de supposer qu'en 1, Pierre quitte le jeu (mais c'est, on le voit, une solution par défaut, puisque aucun coup de Marie n'a alors de sens).

La différence avec l'équilibre obtenu par induction rétrograde, c'est que " Marie quitte en 2 » et "Pierre ne coopère pas en 3 », qui font partie de la solution d'induction rétrograde ou backward induction, ne font pas partie de notre solution, qui se réduit à « Pierre quitte en 1 ", ce qui est cependant une partie commune avec la solution d'induction rétrograde. On évite ainsi le paradoxe. La rationalité n'est pas un fait particulier à chaque joueur, que nous pourrions tester par observation, ce qui conduirait à des observations contradictoires, mais c'est simplement une règle de déduction qui nous permet de définir en chaque nœud le coup choisi. Dans un raisonnement par l'absurde, on maintient toujours les règles de déduction. Simplement, quand une hypothèse nous amène à une contradiction par l'intermédiaire de ces règles, on considère comme valide la négation de l'hypothèse. Et si on est intuitionniste, on introduit à l'inverse la règle de raisonnement par l'absurde comme 
une règle qui, une fois une dérivation menée à l'absurde, élimine la négation accolée à l'hypothèse.

La deuxième différence, c'est que notre raisonnement n'est pas aveugle vers l'arrière et qu'il prend en compte les coups antécédents. Il doit simplement s'assurer que les raisonnements dans le sens passéprésent et ceux dans le sens futur-présent se bouclent bien, c'est-à-dire que lorsqu'on prend un coup présent du raisonnement passé-présent comme antécédent futur dans le raisonnement futur-présent, la conclusion au présent redonne bien le coup correspondant de l'antécédent passé, et réciproquement. On doit donc trouver des valeurs des différents coups possibles qui se redonnent les unes les autres quand on les pose comme données dans les raisonnements passé présent et qu'on les met en boucle avec les raisonnements futurprésent. Ces valeurs sont des points fixes pour cet opérateur qu'est le bouclage des deux raisonnements. Appelons le résultat de ce bouclage l'équilibre épistémique.

Devons-nous en rester là ? Notre raisonnement a pris des coups hypothétiques comme donnés et il en est dérivé des contradictions, si bien que des coups hypothétiques ne pouvaient être maintenus. Il restait alor's plusieurs possibilités : soit qu'en ce noud un autre coup ait été choisi, soit qu'on n'ait pas atteint ce noud. D'autres raisonnements par l'absurde ont tranché entre ces possibilités. Mais l'important, c'est que nous ayons raisonné sur des coups supposés donnés, en prenant nos décisions d'action comme conclusions. Or, dans un jeu, rien n'est donné, sinon la structure du jeu et ses gains (ou du moins l'ordre relatif des gains). Les coups des autres ne sont pas donnés, ils peuvent changer, puisque ce sont des actions à choisir et qu'une première approche naturelle des actions, c'est de les considérer comme des changements du monde. Nous avons donc maintenant, grâce au raisonnement d’induction rétrograde, un monde de référence, mais nous devons examiner ce qui se passe sur des mondes qui impliquent des changements par rapport à ce monde. Ces mondes où des changements se sont produits sont, en un sens, le même monde que le nôtre, mais on y tient compte de ce que les autres ont pu agir, donc y introduire des changements. Nous envisageons ainsi comme hypothèses des changements par rapport aux actions que nous avons supposées données. Il nous faut ensuite décider quelles actions nous choisirions si ces changements avaient lieu. Nous allons reprendre alors le raisonnement précédent et voir si on peut obtenir un bouclage. Si ce bouclage donne à l'auteur du changement (et bien sûr à celui qui décide de son action en maximisant) un gain supérieur à celui de l'équilibre, nous retiendrons cette stratégie. lci, supposons que Pierre prenne comme hypothèse un changement du coup de Marie en 2 (elle cesse de quitter pour coopérer). Il décide donc de jouer la coopération en 1 et de ne pas coopérer en 3. Marie obtient 1 au lieu de 0 dans l'équilibre précédent et Pierre, 3 au lieu de 1. Ce monde est par conséquent préférable. 
Mais, dira-t-on, pourquoi parier sur des changements que feraient les autres? Après tout, ces changements ne sont pas en notre pouvoir. Et si vraiment nous nous situions dans un monde différent, où les autres auraient accompli ces changements auxquels nous ne pouvons rien, pourquoi tenir compte du résultat du premier monde, celui où nous prenons les actions des autres comme données? Dès lors, Marie, supposant que Pierre a coopéré, choisit de quitter et ce monde ne sera pas l'un des préférés de Pierre!

Certes, chacun raisonnant de son côté, sans communiquer, il ne nous est pas possible de changer les actions d'autrui par une sorte de télépathie active. Mais il nous est possible, à l'inverse, d'envisager des changements de notre côté et de nous demander ce que ferait autrui s'il envisageait ces changements. Au lieu de prendre pour antécédent de notre raisonnement le changement d'autrui, ce qui peut apparaittre comme une hypothèse gratuite, nous prenons pour antécédent notre propre changement et nous considérons s'il ne serait pas rationnel pour autrui de changer. Ainsi, Pierre peut coopérer en 1 parce que, suppose-t-il, si Marie envisageait ce changement, elle coopérerait, ce qui donnerait à Pierre son gain maximum en 3. Mais encore une fois, pourquoi, si Pierre coopère en 1, Marie ne quitterait-elle pas en 2 ?

Parce qu'un tel monde ne permettrait pas d'obtenir la stabilité dans le raisonnement en boucle que nous avons déjà fait. Si l'on veut. la défection de Marie consisterait pour elle à changer de monde et à revenir dans le monde de départ. Or, dans le seul monde de départ stable, elle n'obtient que 0 .

Mais que serait un bouclage différent? Ce serait justement un bouclage où, à un changement de Marie pris pour antécédent (au futur) du raisonnement de Pierre, correspondrait un changement de Pierre pris pour antécédent (au passé) du raisonnement de Marie. Mais ici, puisque nous parlons de changements et que chacun est à l'initiative d'un changement qui ne se justifie que s'il redonne le changement de l'autre, ce que nous appelions d'abord le raisonnement de Pierre est justement celui que fait Marie, et réciproquement. Le changement que Marie tente, une fois pris comme antécédent (futur), ne produit pas immédiatement comme conclusion le changement de Pierre, il la produit si le changement de Pierre en question, une fois pris comme antécédent (passé), a pour conclusion le changement de Marie qui, une fois pris comme antécédent (futur), etc. Bref, le raisonnement en boucle se mord la queue puisque dans la dérivation qui justifie chaque raisonnement, nous devons retrouver incluse la boucle qui assure qu'on est à un équilibre. Mais ce raisonnement en boucle n'a rien de vicieux. Il suffit que présupposer un changement de Marie permette de justifier un changement présupposé de Pierre, et réciproquement, pour que la boucle soit stable. Au lieu de trouver une valeur donnée du coup de Marie qui, étant donnée, redonne le coup de Pierre qui la rend possible, nous devons à la fois trouver deux valeurs, du changement 
de Marie et du changement de Pierre, qui, étant données, justifient le bouclage entre ces deux changements. Au lieu d'avoir une série de coups séquentiels qui se présupposent et se justifient les uns les autres, nous avons des séries entrelacées qui se présupposent et se justifient. Nous n'avons fait qu'étendre la notion d'équilibre. Dans chaque raisonnement, la conclusion de l'autre n'est donnée qu'une fois trouvés l'équilibre et le bouclage. Au lieu que l'équilibre soit la conséquence d'une rationalité instrumentale qui prend l'action de l'autre pour donnée, il est devenu un outil de dérivation, comme l'est aussi la maximisation. La différence entre la rationalité instrumentale et la rationalité interactive, c'est que dans la première, la maximisation intervient avant la considération de l'équilibre et indépendamment de celle-ci, et que dans la seconde, elle intervient en s'entrelaçant avec celle de l'équilibre. Nous nommerons alors cela un équilibre interactif.

Comment intervient la rationalité interactive? Elle intervient d'abord pour justifier la tentative de changement. Une fois parvenue à l'équilibre épistémique, Marie se dit qu'elle a abandonné la possibilité d'avoir 1 au lieu de 0 , une fois que son coup maximisateur à court terme (« quitter en $2 »$ ) s'est trouvé défait par le coup de Pierre en 1. Et de même, mutatis mutandis, pour Pierre. On compare ici les gains possibles sur d'autres voies à ceux de l'équilibre. On introduit ensuite la procédure de recherche de l'équilibre interactif' en tentant des changements qui puissent s'appuyer l'un sur l'autre et donc, se boucler. Ainsi, en supposant que Pierre ait changé pour a coopérer en 1 ", un coup de Marie pour " quitter en 2 s ne produit pas de tel bouclage (or, on pourrait le considérer comme un changement, puisque l'équilibre épistémique nous donne «Marie ne joue pas en $1 \%$. La recherche du bouclage semble l'emporter ici sur la maximisation, mais c'est une apparence : en fait, ce coup de Marie interdit le bouclage interactif et nous renvoie justement comme seul bouclage à celui de l'équilibre épistémique, dont on tentait de sortir, ce qui était justifié par des considérations de maximisation. Enfin, une fois qu'on a trouvé un bouclage, il n'est validé que si les gains pour tous ceux qui ont changé sont supérieurs à ceux obtenus dans l'équilibre épistémique. La maximisation a donc d'abord un rôle heuristique, puis le bouclage a un rôle sélectif, mais cette sélection n'est validée que par la maximisation.

L'esprit de la rationalité interactive est simple. Une fois défini un équilibre épistémique qui est instrumental, nous explorons les mondes différents que l'on pourrait atteindre par des changements. Mais ces changements ne peuvent pas être simplement posés par hypothèses comme des données, car cela serait supposer qu'un deus ex machina nous a transportés dans ces mondes. Or, si nous pouvons envisager que le monde réel change sans que nous y soyons pour rien, nous ne pouvons pas supposer que le monde d'un jeu change sans que les joueurs y soient pour rien. Il faut donc que ces 
changements soient des actions des joueurs. Mais ce sont des actions non pas pour répondre à des données du monde, mais pour changer de monde, et dans ce transfert chaque joueur doit s'appuyer sur des changements des autres puisque l'équilibre épistémique nous garantissait qu'aucun joueur n'avait intérêt à en changer unilatéralement. Nous avons cependant, en envisageant des changements de monde, dissipé l'apparence de finalisme de ces changements, qu'on pourrait croire introduits pour obtenir des autres qu'ils changent. Nous devons simplement considérer des changements qui font changer de monde chacun des joueurs (par rapport à l'équilibre épistémique). Une fois ces changements supposés, nous vérifions s'ils donnent lieu au bouclage interactif décrit plus haut. Les changements en question sont done simplement ceux qui ont la propriété, une fois introduits comme antécédents des raisonnements par chacun des joueurs, de produire ce bouclage. Ce bouclage nous permet le changement de monde. Une fois ce changement de monde possible, nous sélectionnons un monde par maximisation par rapport à l'équilibre épistémique, puis entre les équilibres interactifs s'ils sont plusieurs. Nous transformons donc la finalité apparente en un raisonnement à la fois causal et sélectif. Les changements envisagés ont la propriété de déclencher soit des instabilités, soit des bouclages, quand ils s'appuient les uns sur les autres. L'équilibre épistémique sélectionnait les actions qui étaient stables par rapport à des coups supposés donnés. L'équilibre interactif sélectionne des actions qui sont stables par rapport à des actions qui les présupposent pour être stables. C'est une propriété contextuelle de ces actions, le contexte étant donné par les autres actions. Il n'y a pas là de finalité, ni de télépathie active entre les joueurs, puisque chacun peut vérifier cette propriété de son côté.

Mais qu'en est-il du dilemme du prisonnier (si Marie coopère et Pierre coopère, cela leur donne 2 et 2 ; si Marie fait défection et Pierre fait défection, cela leur donne 1 et 1 ; celui qui coopère quand l'autre fait défection a 0 et l'autre 3 ) ? Le problème est que dans ce jeu Marie, par exemple, doit envisager, quand elle joue, deux possibilités en parallèle, celle que Pierre a coopéré ou celle qu'il ait fait défection, et que son coup doit répondre à ces deux coups possibles. Dans notre raisonnement en boucle, il faut donc envisager deux boucles. Si elle change pour la coopération, le Pierre qui a coopéré s'en réjouit, mais aussi, sur l'autre boucle, celui qui a fait défection, et il s'en réjouit même davantage, si bien que Pierre préfère toujours faire défection. Si une des boucles est stable, l'autre ne l'est pas, à première vue.

En fait, là aussi il faut que les changements s'appuient l'un sur l'autre, si bien qu'un changement de Marie doit s'appuyer sur un changement de Pierre, et réciproquement. Il faut donc que les changements aient lieu dans les deux boucles. C'est dire que le changement de Marie pour la coopération doit s'appuyer dans une 
boucle sur le retour de Pierre à la coopération qu'il avait d'abord envisagée puis trouvée instable, et dans l'autre sur' son changement pour la coopération, à partir d'une défection qui s'était trouvée stable dans l'équilibre épistémique. Et le raisonnement inverse se fait pour Pierre. La bizzarerie qui consiste à devoir imaginer des changements dans plusieurs mondes à la fois tient simplement à ce qu'aucun des acteurs ne peut distinguer entre ces mondes (puisque l'incertitude de Marie est en fait aussi partagée par Pierre).

Le partisan de la pure rationalité instrumentale, qui ne raisonne qu'à partir du donné, n'admettra pas cette rationalité interactive qui bâtit des échafaudages s'appuyant non pas sur le sol, mais les uns sur les autres. Par ailleurs, il est curieux. qu'il accepte de raisonner sur les jeux évolutionnaires, où on se donne des mutations, c'est-à-dire la possibilité de changements, qui ne sont finalement justifiés que parce qu'ils donneront lieu à une diffusion en s'appuyant sur les conflits des sous-populations en présence, conflits dont l'issue ne tient pas à la valeur d'une stratégie dans l'absolu, mais seulement à la valeur de cette stratégie relativement aux stratégies en présence. Et ce que montre la théorie des jeux évolutionnaires, quand elle conduit parfois à conserver des stratégies faiblement dominantes, c'est que justement le raisonnement qui dit a supposé donné $x$, je choisis $z$, supposé donné non $\mathrm{x}$, je choisis $\mathrm{z}$, donc je choisis $\mathrm{z}$ ", raisonnement qui définit $z$ comme stratégie dominante, n'est pas le schéma que suit l'évolution dans les jeux. L'évolution permet de se libérer d'une simple rationalité instrumentale, puisqu'elle introduit des mutations qui n'étaient pas données initialement et qu'elle joue sur les capacités relatives des stratégies, sur leur succès dans l'interaction. Mais elle ne va pas jusqu'à privilégier cette interaction, ce que semblent pouvoir faire les humains.

On voit donc que le double pessimisme de Binmore n'était pas justifié quand il pensait que le paradoxe de l'induction rétrograde condamnait l'approche raisonneuse et que les jeux évolutionnaires nous ouvraient des domaines nouveaux (comprenant des équilibres autres que ceux de Nash), alors que l'approche raisonneuse était désormais stérile. On peut dénouer le paradoxe apparent et on peut trouver de nouveaux équilibres, autres que les équilibres de Nash, en demeurant dans l'approche raisonneuse. Il faut simplement faire des raisonnements complets qui ne soient pas aveugles aux coups antécédents ou encore qui ne traitent pas les actions comme de simples données. Bref, il faut accepter de pleinement développer la notion de rationalité interactive, en acceptant de considérer des changements du monde hypothétiques et des changements interactifs, c'est-à-dire des actions qui ont pour fonctions réciproques, pour effets conditionnés par une cause qui, sur une autre ligne, est l'effet de leur causalité, de justifier des changements des actions des autres. 
Mais cette extension nous amène à suivre Binmore dans sa volonté de sortir du cadre étroit du a bayésianisme ". Une révision "bayésienne " consiste à partir d'une partition des états du monde, qu'on range en quelque sorte dans des cases à chacune desquelles on assigne une probabilité. Les informations nouvelles nous obligent souvent à changer la taille respective de ces cases, c'est-à-dire à modifier les probabilités. Mais que faire d'un événement qui ne figurait pas dans nos cases? La révision bayésienne ne nous le dit pas. L'objection de Binmore (1993) est alors que cette révision ne peut modéliser un processus d'apprentissage. Un véritable apprentissage ne consiste pas, en effet, à simplement ajuster les tailles respectives des cases de notre savoir, mais bien à créer d'autres cases, à remodeler les anciennes en les fusionnant ou en les divisant.

Dans le raisonnement de la théorie des jeux, on peut introduire de l'incertitude et probabiliser les coups. Mais si on suit le raisonnement d'induction rétrograde, le seul qui puisse utiliser la notion de révision bayésienne, on devra alors en rester aux cases qui résistent au premier raisonnement par l'absurde et considérer que les autres coups ne figurent tout simplement pas dans nos cases. Or, le raisonnement actionnel, qui suppose que le monde puisse avoir changé, et le raisonnement interactif, qui introduit des changements susceptibles d'en obtenir d'autres, exigent de considérer d'autres cases. Il faut donc utiliser d'autres types de révision. Le premier raisonnement continue à utiliser une notion de révision épistémique, qui prend l'information pour donnée, et met les actions en cohérence avec cette information. Mais le second raisonnement doit introduire une notion de révision actionnelle qui est partiellement formalisée dans la notion de mise à jour (updating) de Katsuno et Mendelzon. Dans cette révision, on doit envisager en parallèle les différents mondes, celui dans lequel il n'y a pas eu de changement et ceux dans lesquels il y en a eu. La rationalité interactive exige au moins cette révision actionnelle.

Revenons pour finir aux jeux évolutionnaires. Nous pouvons maintenant noter les relations utiles entre les deux approches, raisonneuse et évolutionnaire. Les dynamiques populationnelles ont l'intérêt de nous donner une idée du résultat des opérations d'une rationalité actionnelle et d'une rationalité interactive. Elles proposent bien chaque action d'un type d'individu, donc chaque changement du monde, comme un contexte pour les actions des autres. Elles mettent bien en scène une histoire qui va revenir sur certaines stratégies qui semblaient condamnées dans un premier temps, pour finalement leur donner la prédominance, et ce, grâce au conflit qui existe entre des stratégies temporairement dominantes (des équilibres temporaires). On peut donc y trouver une situation analogue à celle où l'on change de stratégie pour justifier des changements de stratégie chez les autres, ou encore à ce raisonnement qui envisage successivement différents types d'équilibre. Plus exactement, la 
stratégie dominante va changer et les stratégies auxquelles la nouvelle stratégie dominante sera confrontée vont changer en même temps.

Mais la différence essentielle, c'est que les jeux évolutionnaires ne proposent pas de révision. Ou plus exactement, c'est la dynamique qui impose la révision, ce ne sont pas les acteurs qui la font. Or, l'intérêt de l'approche raisonneuse, c'est justement de pouvoir faire la révision avant même d'avoir agi, c'est de pouvoir faire des révisions virtuelles, pour finalement choisir la révision dominante. Mais c'est seulement à condition de concevoir cette révision comme actionnelle et non pas comme épistémique, comme produisant un changement de contexte et non pas comme tenant compte passivement d'une donnée, que l'on peut échapper aux impasses de la théorie raisonneuse. Les jeux évolutionnaires sont une façon pour le modélisateur de retrouver cette richesse qu'il doit bien donner à l'acteur de la théorie des jeux, sans justement avoir à partager cette richesse avec ses joneurs. Les jeux évolutionnaires supposent données les différentes mutations et populations, ainsi que les dynamiques de mutations ou réplication. Ils ne les font pas émerger des raisonnements actionnels ou interactifs de leurs joueurs. Ils échappent aux problèmes de la perspective raisonneuse simplement parce qu'ils ne se les posent pas. Supposer les choses données, c'est donc l'intérêt des jeux évolutionnaires (et c'est bon pour la théorie de l'évolution, qui est une explication après coup). On comprend que la théorie des jeux évolutionnaires séduise les théoriciens des jeux et les économistes, accoutumés à tenir le rôle du modélisateur en se construisant des acteurs sur mesure. Ils peuvent ainsi explorer à loisir les évolutions complexes de ces dynamiques populationnelles, y compris en recourant à des simulations quand la compréhension analytique est hors de portée. Mais notre rationalité interactive produit aussi une autre sorte de complexité qui consiste précisément à anticiper sur ce qui n'est pas donné et à réviser nos actions de telle façon que les autres aient raison de réviser les leurs.

Département de philosophie Aix-Marseille I 